\title{
A Parallel Splitting up Algorithm for the Determination of an Unknown Coefficient in Multi Dimensional Parabolic Problem
}

\author{
Daoud S. Daoud and D. Subasi \\ Department of Mathematics, Eastern Mediterranean Univ., Famaqusta, North \\ Cyprus - Mersin 10 Turkey \\ daoud.daoud@emu.edu.tr
}

\begin{abstract}
One of the global approach for solving the two dimensional inverse parabolic problem is the predictor corrector which takes place for evaluating the pair $(u, p)$ and adjusting the evaluation for the desired accuracy. In this work we will present a new parallel algorithm(of non iterative type) for solving two or higher dimensional inverse control problem.
\end{abstract}

\section{Introduction}

In this article we study the finite difference method for approximating the source control parameter $p(t)$, and the solution $u=u(x, y, t)$ of the following inverse model problem defined by; Find $u=u(x, y, t)$ and $p=p(t)$ which satisfy

$$
\begin{aligned}
& u_{t}=\quad \Delta u+p(t) u+\phi(x, y, t) \text { in } \Omega \times[0, T] \\
& u(x, y, 0)=f(x, y)(x, y) \in \Omega \\
& u(x, y, t)=g(x, y, t) \quad \text { on } \partial \Omega \times[0, T]
\end{aligned}
$$

subject to the over specification condition at a point $\left(x^{*}, y^{*}\right)$ in the spatial domain $\Omega$ given by;

$$
u\left(x^{*}, y^{*}, t\right)=E(t)
$$

One of the global techniques for solving problem (11) and (2) is the predictor corrector finite difference scheme introduced by J.R. Canon et al. [1].

The proposed splitting algorithm is defined to split the problem in accordance to the spatial variables into independent one dimensional sub problems which classified as Additive Splitting Method and its of non iterative type.

\section{$2 \quad$ Parallel Splitting up Method}

The general definition of the multi dimensional parabolic problem is given by

$$
\frac{\partial u}{\partial t}=\sum_{j}^{m} \frac{\partial}{\partial x_{j}}\left(a_{j} \frac{\partial u}{\partial x_{j}}\right)+f \quad \text { in } \Omega \times[0, T],
$$


where $u(0)=u_{0}$, with Dirichlet boundary conditions over $\partial \Omega \times[0, T]$.

The domain $\Omega$ is a bounded domain in $R^{m}(m=2,3), a_{j}$ are scalars, and the assembled coefficients of the central difference discretization for $\frac{\partial}{\partial x_{j}}\left(a_{j} \frac{\partial u}{\partial x_{j}}\right)$, and for $\sum_{j=1}^{m} \frac{\partial}{\partial x_{j}}\left(a_{j} \frac{\partial u}{\partial x_{j}}\right)$ are denoted by $A_{j}$ and $A$, respectively. The key to the splitting algorithm is the splitting up algorithm by Lu, et. al. [3], given by

Algorithm 1 First order implicit splitting up method.

Step1. Let $\delta t>0$ be any time step. For $j=1, \ldots m$ the solution $v^{n+\frac{j}{2 m}}$ is given by:

$$
\left(I-m \delta t A_{j}\right) v^{n+\frac{j}{2 m}}=v^{n}+m \delta t f^{n+1}
$$

Step 2. $v^{n+1}=\frac{1}{m} \sum_{j=1}^{m} v^{n+\frac{j}{2 m}}$.

Step 3. If $T<(n+1) \delta t$ go to step 1, otherwise stop.

Theorem 2. The splitting up method given by algorithm 1 is globally a first order splitting in $\delta t$.

Proof. For the proof see [2]

Theorem 3. With respect to each spatial variable $x_{j}, j=1, \ldots m$ the solution $v^{n+\frac{j}{2 m}}$ given by step 1, and the solution $v^{n+1}$ by step 2 of algorithm 1 is unconditionally stable in $\ell_{2}$ norm.

Proof. For the proof see [2]

\section{Inverse Control Problem Splitting Algorithm}

In this section we will present the parallel splitting up algorithm for the inverse control problem (1+2).

Algorithm 4 Inverse Problem Splitting up Method.

Step1. Let $\delta t>0$ be any time step. For $j=1, \ldots m$ solve for $v^{n+\frac{j}{2 m}}$ :

$$
\frac{v^{n+j / 2 m}-v^{n}}{m \delta t}=A_{j} v^{n+j / 2 m}+\frac{1}{m} p^{n+1} v^{n+j / 2 m}+\frac{1}{m} \phi^{n+1}
$$

Step 2. $v^{n+1}=\frac{1}{m} \sum_{j=1}^{m} v^{n+\frac{j}{2 m}}$.

Step 3. If $T<(n+1) \delta$ t go to step 1, otherwise stop.

In the predictor corrector algorithm we firstly assume that $v(x, y, t)$ and $p(t)$ are given at the time $t=n \delta t$, for $\delta t>0$, and let $l=0,1, \ldots$ be the correction level. The generic splitting up and Predictor-Corrector algorithm is given by

Algorithm 5 Predictor Corrector Method for Inverse Control Problem

Step 1: For $l=0$ let $p^{n+1(l)}=p^{n}$, solve the following splitted one dimensional problem

$$
\begin{aligned}
& \frac{v^{n+1 / 4}-v^{n}}{2 \delta t}=A_{x} v^{n+1 / 4}+\frac{1}{2} p^{n+1(0)} v^{n+1 / 4}+\frac{1}{2} \phi^{n+1}, \\
& \frac{v^{n+1 / 2}-v^{n}}{2 \delta t}=A_{y} v^{n+1 / 2}+\frac{1}{2} p^{n+1(0)} v^{n+1 / 2}+\frac{1}{2} \phi^{n+1},
\end{aligned}
$$


the solution $v^{n+1(l)}=\frac{1}{2}\left(v^{n+1 / 4}+v^{n+1 / 2}\right)$, where $A_{x}$, and $A_{y}$ represent the assembled coefficients of the central difference approximation for $u_{x x}$, and $u_{y y} r e-$ spectively.

Step 2: Since $v\left(x_{i^{*}}, y_{j^{*}}, t\right)=E(t)$, where $\left(x_{i^{*}}, y_{j^{*}}\right)=$ $\left(x^{*}, y^{*}\right)$ i.e $v\left(x_{i^{*}}, y_{j^{*}},\right)^{n+1(l)}=E^{n+1(l)}$, then problem (11) for $E(t)$ is given by

$$
E^{\prime}(t)=\triangle v\left(x_{i^{*}}, y_{j^{*}}, t\right)+p(t) E(t)+\phi\left(x_{i^{*}}, y_{j^{*}}, t\right),
$$

then the splitting up of (7) in accordance to the splitting up algorithm is given by

$$
\begin{aligned}
& \frac{E^{n+1 / 4(l)}-E^{n}}{2 \delta t}=v_{x x}\left(x_{i^{*}}, y_{j^{*}}, .\right)^{n+1(l)}+\frac{1}{2} p x^{n+1(l+1)} E^{n+1 / 4(l)}+\frac{1}{2} \phi^{n+1}, \\
& \frac{E^{n+1 / 2(l)}-E^{n}}{2 \delta t}=v_{y y}\left(x_{i^{*}}, y_{j^{*}}, .\right)^{n+1(l)}+\frac{1}{2} p y^{n+1(l+1)} E^{n+1 / 2(l)}+\frac{1}{2} \phi^{n+1} .
\end{aligned}
$$
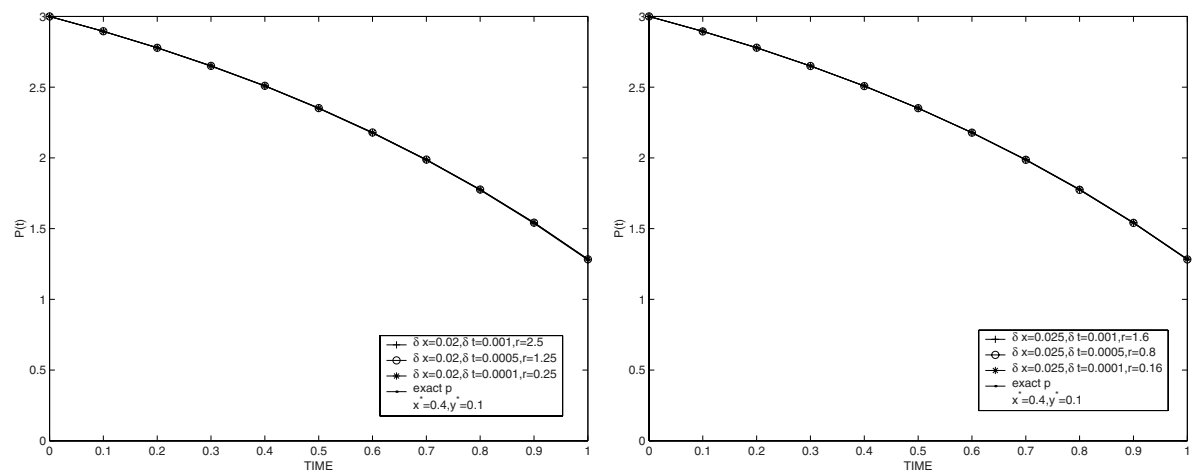

Fig. 1. The exact value and the estimated values of $p(t)$ for different $\delta x$ and $\delta t$ for problem 1 , simulated over $[0,1]$

Table 1. The error due to the solution of problem 1 for $x^{*}=0.4$ and $y^{*}=0.1$

\begin{tabular}{|c|ccccc|}
\hline & \multicolumn{5}{|c|}{$\delta x=0.02$} \\
\hline$r$ & 2.5 & 1.6 & 1.25 & 0.8 & 0.25 \\
\hline error & $4.63008 \mathrm{e}(-4)$ & $2.52485 \mathrm{e}(-4)$ & $2.07695 \mathrm{e}(-4)$ & $8.93606 \mathrm{e}(-5)$ & $6.05821 \mathrm{e}(-5)$ \\
\hline
\end{tabular}

The solution of (8) for $p x$ and py is given by

$$
\begin{gathered}
p x^{n+1(l+1)}=\frac{\left(E^{n+1 / 4(l)}-E^{n}\right)-2 \delta t v_{x x}\left(x_{i^{*}}, y_{j^{*}}, .\right)^{n+1(l)}-\delta t \phi^{n+1}}{\delta t E^{n+1 / 4(l)}}, \\
p y^{n+1(l+1)}=\frac{\left(E^{n+1 / 2(l)}-E^{n}\right)-2 \delta t v_{y y}\left(x_{\left.i^{*}, y_{j}, .\right)^{n+1(l)}-\delta t \phi^{n+1}}^{\delta t E^{n+1 / 2(l)}},\right.}{p^{n+1(l+1)}=\frac{1}{2}\left(p x^{n+1(l+1)}+p y^{n+1(l+1)}\right),}
\end{gathered}
$$


where $p x$ and py represent the correction with respect to $x$ and $y$ variables respectively.

Step3: Update the solution $v^{n+1(l)}$ using step 1 for $l=1,2, \ldots$ and considers the latest value of $p^{n+1(l+1)}$.

The value of $p^{n+1(l+1)}$ will then be adjusted till it satisfies the pre assigned tolerance(e.g. $\left.\left|p^{n+1(l+1)}-p^{n+1(l)}\right| \leq 10^{-4}\right)$ for the evaluation of the pair $(v, p)$ over the time interval $\left[t_{n}, t_{n+1}\right]$.

Theorem 6. The splitting up algorithm 4 of the inverse problem is a globally a first order algorithm in $\delta t$.

\section{Numerical Experiments and Conclusion}

In this section we report the numerical results from solving an inverse control problem using algorithm 5 described in section 3 for evaluating the pair $(u, p)$. In general we take an exact solution $u(x, y, t)$, source function $p(t)$ we refer to the general problem (1)-(2) to formulate our model problems using algorithm 5 to compute the value of the solution $v^{n+1}$ and $p(t)$, and to compare the numerical results with the exact values.

Model problem 1. We consider the following problem

$$
u_{t}=\Delta u+p(t) u+\phi(x, y, t),
$$

defined over $\Omega \times[0,1]$ with initial conditions $u(x, y, 0)=\sin \left(\frac{\pi}{4}(x+2 y)\right)$ and the boundary conditions given by

$$
\begin{aligned}
& u(0, y, t)=e^{t} \sin \left(\frac{\pi}{2} y\right), u(1, y, t)=e^{t} \sin \left(\frac{\pi}{4}(1+2 y)\right), \\
& u(x, 0, t)=e^{t} \sin \left(\frac{\pi}{4} x\right), u(x, 1, t)=e^{t} \sin \left(\frac{\pi}{4}(x+2)\right),
\end{aligned}
$$

From the numerical results we demonstrate the accuracy of the evaluated function $p(t)$ over the whole time interval $[0,1]$ independently on the time stepping or the spatial mesh spacings.

\section{References}

1. J.R. Cannon, Yanping Lin, S.Wang, Determination of source parameter in parabolic equations. Meccanica, vol. 27:85-94,1992.

2. D.S. Daoud, Non Overlapping Domain Decomposition and Additive Splitting up Methods for Multi Dimensional Parabolic Problem, Numer. Methods Partial Differerntial Eq. to appear.

3. T.Lu, P. Neittaanmaki, and X-C Tai, A parallel splitting up method and its application to Navier-Stokes equations. Applied Mathematics Letters, Vol. 4, No. 2:25-29, 1991. 